\title{
Some inequalities for nonnegative tensors
}

\author{
Jun He* ${ }^{*}$ Ting-Zhu Huang and Guang-Hui Cheng
}

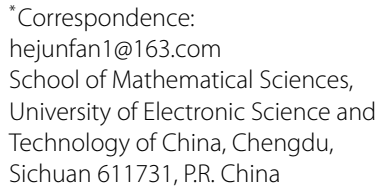

\begin{abstract}
Let $\mathcal{A}$ be a nonnegative tensor and $x=\left(x_{i}\right)>0$ its Perron vector. We give lower bounds for $x_{t}^{m-1} / \sum x_{i_{2}} \cdots x_{i_{m}}$ and upper bounds for $x_{s}^{m-1} / \sum x_{i_{2}} \cdots x_{i_{m}}$, where $x_{s}=\max _{1 \leq i \leq n} x_{i}$ and $x_{t}=\min _{1 \leq i \leq n} x_{i}$.
\end{abstract}

MSC: $15 \mathrm{~A} 18 ; 15 \mathrm{~A} 69 ; 65 \mathrm{~F} 15 ; 65 \mathrm{~F} 10$

Keywords: Perron vector; nonnegative tensor; spectral radius; eigenvalues

\section{Introduction}

Eigenvalue problems of higher order tensors have become an important topic of study in a new applied mathematics branch, numerical multilinear algebra, and they have a wide range of practical applications [1-7]. The main difficulty in tensor problems is that they are generally nonlinear. Therefore, large amounts of results for matrices are never in force for higher order tensors. However, there are still some results preserved in the case of higher order tensors.

Throughout this paper we consider an $m$ th order $n$-dimensional tensor $\mathcal{A}$ consisting of $n^{m}$ entries in $\mathbb{R}$ :

$$
\mathcal{A}=\left(a_{i_{1}, i_{2}, \ldots, i_{m}}\right), \quad a_{i_{1}, i_{2}, \ldots, i_{m}} \in \mathbb{R}, 1 \leq i_{1}, i_{2}, \ldots, i_{m} \leq n
$$

The tensor $\mathcal{A}$ is called nonnegative (or positive) if all the entries $a_{i_{1}, i_{2}, \ldots, i_{m}} \geq 0$ (or $a_{i_{1}, i_{2}, \ldots, i_{m}}>$ $0)$. We also denote by $\mathbb{C}$ the field of complex numbers.

Definition 1.1 A pair $(\lambda, x) \in \mathbb{C} \times \mathbb{C}^{n} /\{0\}$ is called an eigenvalue-eigenvector pair of $\mathcal{A}$, if they satisfy

$$
\mathcal{A} x^{m-1}=\lambda x^{[m-1]},
$$

where $n$-dimensional column vectors $\mathcal{A} x^{m-1}$ and $x^{[m-1]}$ are defined as

$$
\mathcal{A} x^{m-1}:=\left(a_{i i_{2} \cdots i_{n}} x_{i_{2}} \cdots x_{i_{n}}\right)_{1 \leq i \leq n} \quad \text { and } \quad x^{[m-1]}:=\left(x_{i}^{m-1}\right)_{1 \leq i \leq n} .
$$

This definition was introduced by Qi [8] when $m$ is even and $\mathcal{A}$ is symmetric. Independently, Lim [9] gave such a definition but restricted $x$ to be a real vector and $\lambda$ to be a real number. Let

$$
\rho(\mathcal{A})=\max \{|\lambda|: \lambda \text { is an eigenvalue of } \mathcal{A}\},
$$

where $|\lambda|$ denotes the modulus of $\lambda$. We call $\rho(\mathcal{A})$ the spectral radius of tensor $\mathcal{A}$.

\section{Springer}

○2014 He et al.; licensee Springer. This is an Open Access article distributed under the terms of the Creative Commons Attribution License (http://creativecommons.org/licenses/by/2.0), which permits unrestricted use, distribution, and reproduction in any medium, provided the original work is properly cited. 
In [4], Chang et al. generalized the Perron-Frobenius theorem from nonnegative matrices to irreducible nonnegative tensors. Some further results based on this theorem are discussed by Yang $[10,11]$.

Definition 1.2 The tensor $\mathcal{A}$ is called reducible if there exists a nonempty proper index subset $\mathbb{J} \subset\{1,2, \ldots, n\}$ such that

$$
a_{i_{1}, i_{2}, \ldots, i_{m}}=0, \quad \forall i_{1} \in \mathbb{J}, \forall i_{2}, \ldots, i_{m} \notin \mathbb{J} .
$$

If $\mathcal{A}$ is not reducible, then we call $\mathcal{A}$ irreducible.

Theorem 1.3 [4] If $\mathcal{A}$ is irreducible and nonnegative, then there exist a number $\rho(\mathcal{A})>0$ and $a$ vector $x_{0}>0$, such that

$$
\mathcal{A} x_{0}^{m-1}=\rho(\mathcal{A}) x_{0}^{[m-1]} .
$$

Moreover, if $\lambda$ is an eigenvalue with a nonnegative eigenvector, then $\lambda=\rho(\mathcal{A})$. If $\lambda$ is an eigenvalue of $\mathcal{A}$, then $|\lambda| \leq \rho(\mathcal{A})$.

We call $x_{0}$ a Perron vector of $\mathcal{A}$ corresponding to its largest nonnegative eigenvalue $\rho(\mathcal{A})$.

In this paper, we are interested in studying some bounds for the Perron vector of $\mathcal{A}$. For this purpose, we define

$$
x_{s}=\max _{1 \leq i \leq n} x_{i}, \quad x_{t}=\min _{1 \leq i \leq n} x_{i} .
$$

In the following we first give a new and simple bound for $x_{s} / x_{t}$. Then we give some lower bounds for $x_{t}^{m-1} / \sum x_{i_{2}} \cdots x_{i_{m}}$ and upper bounds for $x_{s}^{m-1} / \sum x_{i_{2}} \cdots x_{i_{m}}$, which can be used to get another bound for $x_{s} / x_{t}$.

The paper is organized as follows. In Section 2, some efforts of establishing the bounds of the nonnegative tensor are made. An application of these bounds is studied in Section 3.

\section{Bounds}

In [12], the authors have studied the perturbation bound for the spectral radius of $\mathcal{A}$, and they show that a Perron vector $x$ must be known in advance so that the perturbation bound can be computed. Here we cite a lemma for use below.

Lemma 2.1 [12] Suppose $\mathcal{A}$ is nonnegative such that $m \geq 3$ and $x$ is its Perron vector $x$. Then

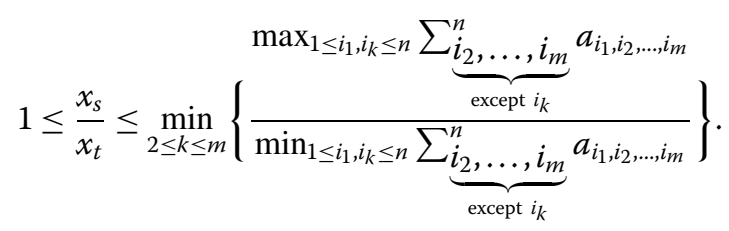


Define the $i$ th row sum of $\mathcal{A}$ as

$$
R_{i}(\mathcal{A})=\sum_{i_{2}, \ldots, i_{m}=1}^{n} a_{i i_{2} \cdots i_{m}}
$$

and denote the largest, the smallest, and the average row sums of $\mathcal{A}$ by

$$
R_{\max }(\mathcal{A})=\max _{i=1, \ldots, n} R_{i}(\mathcal{A}), \quad R_{\min }(\mathcal{A})=\min _{i=1, \ldots, n} R_{i}(\mathcal{A})
$$

Let

$$
l=\min _{i_{1}, \ldots, i_{m}} a_{i_{1} \cdots i_{m}}, \quad L=\max _{i_{1}, \ldots, i_{m}} a_{i_{1} \cdots i_{m}} .
$$

Theorem 2.2 Suppose $\mathcal{A}$ is nonnegative with Perron vector $x$. Then

$$
\frac{x_{s}}{x_{t}} \geq \max \left\{\left\{\frac{R_{\max }(\mathcal{A})}{R_{\min }(\mathcal{A})}\right\}^{\frac{1}{2(m-1)}}, \max _{2 \leq k \leq m}\left\{\frac{\max _{1 \leq i_{1}, i_{k} \leq n} \sum_{\text {except } i_{k}}^{\sum_{i_{2}, \ldots, i_{m}}^{n}} a_{i_{1}, i_{2}, \ldots, i_{m}}}{\min _{1 \leq i_{1}, i_{k} \leq n} \sum_{\underbrace{i_{2}, \ldots, i_{m}}_{\text {except } i_{k}}}^{i_{i_{1}, i_{2}, \ldots, i_{m}}}}\right\}^{\frac{1}{2 m-3}}\right\} .
$$

Proof Since, for any $i=1,2, \ldots, n$,

$$
\rho(\mathcal{A}) x_{t}^{m-1} \leq \rho(\mathcal{A}) x_{i}^{m-1}=\sum_{i_{2}, \ldots, i_{m}=1}^{n} a_{i i_{2} \cdots i_{m}} x_{i_{2}} \cdots x_{i_{m}} \leq \sum_{i_{2}, \ldots, i_{m}=1}^{n} a_{i i_{2} \cdots i_{m}} x_{s}^{m-1}
$$

we have

$$
\rho(\mathcal{A}) x_{t}^{m-1} \leq R_{\min }(\mathcal{A}) x_{s}^{m-1}
$$

Similarly,

$$
\rho(\mathcal{A}) x_{s}^{m-1} \geq R_{\max }(\mathcal{A}) x_{t}^{m-1}
$$

It follows from (4) and (5) that

$$
\frac{x_{s}^{m-1}}{x_{t}^{m-1}} \geq \frac{x_{t}^{m-1}}{x_{s}^{m-1}} \frac{R_{\max }(\mathcal{A})}{R_{\min }(\mathcal{A})}
$$

and therefore

$$
\frac{x_{s}}{x_{t}} \geq\left\{\frac{R_{\max }(\mathcal{A})}{R_{\min }(\mathcal{A})}\right\}^{\frac{1}{2(m-1)}}
$$


If we assume $\|x\|_{1}=1$, then, for any $i=1,2, \ldots, n$,

$$
\begin{aligned}
\rho(\mathcal{A}) x_{t}^{m-1} & \leq \rho(\mathcal{A}) x_{i}^{m-1}=\sum_{i_{2}, \ldots, i_{m}=1}^{n} a_{i i_{2} \cdots i_{m}} x_{i_{2}} \cdots x_{i_{m}} \\
& \leq\left(\sum_{i_{2}, \ldots, i_{m}=1}^{n} a_{i i_{2} \cdots i_{m}} x_{i_{k}}\right) x_{s}^{m-2} \leq(\min _{1 \leq i_{1}, i_{k} \leq n} \underbrace{\sum_{i_{2}, \ldots, i_{m}}^{n}}_{\text {except } i_{k}} a_{i_{1}, i_{2}, \ldots, i_{m}}) x_{s}^{m-2} .
\end{aligned}
$$

Similarly,

$$
\rho(\mathcal{A}) x_{s}^{m-1} \geq(\max _{1 \leq i_{1}, i_{k} \leq n} \underbrace{\sum_{i_{2}, \ldots, i_{m}}^{n}}_{\text {except } i_{k}} a_{i_{1}, i_{2}, \ldots, i_{m}}) x_{t}^{m-2},
$$

and therefore

$$
\begin{gathered}
\max _{1 \leq i_{1}, i_{k} \leq n} \sum_{x_{t}}^{\sum_{\text {except } i_{k}}^{n}} \geq\left\{\frac{\underbrace{i_{2}, \ldots, i_{m}}_{\text {except } i_{k}}}{\min _{1 \leq i_{1}, i_{k} \leq n} a_{i_{1}, i_{2}, \ldots, i_{m}}^{\sum_{i_{2}, \ldots, i_{m}}^{n}} a_{i_{1}, i_{2}, \ldots, i_{m}}}\right\}^{\frac{1}{2 m-3}} .
\end{gathered}
$$

The result follows.

Remark By the obvious inequality

$$
1 \leq \max \left\{\left\{\frac{R_{\max }(\mathcal{A})}{R_{\min }(\mathcal{A})}\right\}^{\frac{1}{2(m-1)}}, \max _{2 \leq k \leq m}\left\{\frac{\max _{1 \leq i_{1}, i_{k} \leq n} \sum_{\underbrace{i_{2}, \ldots, i_{m}}_{\text {except } i_{k}}}^{n} a_{i_{1}, i_{2}, \ldots, i_{m}}}{\min _{1 \leq i_{1}, i_{k} \leq n} \sum_{\text {except } i_{k}}^{\sum_{i_{2}, \ldots, i_{m}}^{n}} a_{i_{1}, i_{2}, \ldots, i_{m}}}\right\}^{\frac{1}{2 m-3}}\right\}
$$

we see that our new lower bound in (3) is sharper than that in (2).

If the tensor $\mathcal{A}$ is positive, we derive a new upper bound for $x_{s} / x_{t}$ in terms of the entries of $\mathcal{A}$ and the spectral radius $\rho(\mathcal{A})$.

Theorem 2.3 If $\mathcal{A}$ is positive with Perron vector $x$, then

$$
\begin{aligned}
& \frac{l}{\rho(\mathcal{A})-R_{\min }(\mathcal{A})+l(m-1) n} \leq \frac{x_{t}^{m-1}}{\sum_{i_{2}, \ldots, i_{m}=1}^{n} x_{i_{2}} \cdots x_{i_{m}}} \\
& \leq \frac{L}{\rho(\mathcal{A})-R_{\max }(\mathcal{A})+L(m-1) n} \\
& \begin{aligned}
\frac{\rho(\mathcal{A})-R_{\max }(\mathcal{A})+[(m-1) n-1] L}{\rho(\mathcal{A})-R_{\max }(\mathcal{A})+L(m-1) n} \cdot \frac{1}{(m-1) n-1} & \leq \frac{x_{s}^{m-1}}{\sum_{i_{2}, \ldots, i_{m}=1}^{n} x_{i_{2}} \cdots x_{i_{m}}} \\
& \leq \frac{\rho(\mathcal{A})-R_{\min }(\mathcal{A})+l}{\rho(\mathcal{A})-R_{\min }(\mathcal{A})+l(m-1) n}
\end{aligned}
\end{aligned}
$$


Proof First, we prove the right side of (9). Now we consider

$$
\begin{aligned}
\rho(\mathcal{A}) x_{t}^{m-1}-l \sum_{i_{2}, \ldots, i_{m}=1}^{n} x_{i_{2}} \cdots x_{i_{m}} & =\sum_{i_{2}, \ldots, i_{m}=1}^{n} a_{t i_{2} \cdots i_{m}} x_{i_{2}} \cdots x_{i_{m}}-l \sum_{i_{2}, \ldots, i_{m}=1}^{n} x_{i_{2}} \cdots x_{i_{m}} \\
& \geq x_{t}^{m-1}\left[R_{\min }(\mathcal{A})-l(m-1) n\right]
\end{aligned}
$$

and

$$
\begin{aligned}
\frac{x_{s}^{m-1}}{\sum_{i_{2}, \ldots, i_{m}=1}^{n} x_{i_{2}} \cdots x_{i_{m}}} & =1-\frac{\sum_{\begin{array}{c}
i_{2}, \ldots, i_{m}=1 \\
\text { except } i_{2}=\cdots=i_{m}=s
\end{array}}^{n} x_{i_{2}} \cdots x_{i_{m}}}{\sum_{i_{2}, \ldots, i_{m}=1}^{n} x_{i_{2}} \cdots x_{i_{m}}} \\
& \leq 1-\frac{[(m-1) n-1]}{\sum_{i_{2}, \ldots, i_{m}=1}^{n} x_{i_{2}} \cdots x_{i_{m}}} x_{t}^{m-1},
\end{aligned}
$$

so we can get

$$
\begin{aligned}
\frac{x_{t}^{m-1}}{\sum_{i_{2}, \ldots, i_{m}=1}^{n} x_{i_{2}} \cdots x_{i_{m}}} & \geq \frac{l}{\rho(\mathcal{A})-R_{\min }(\mathcal{A})+l(m-1) n}, \\
\frac{x_{s}^{m-1}}{\sum_{i_{2}, \ldots, i_{m}=1}^{n} x_{i_{2}} \cdots x_{i_{m}}} & \leq \frac{\rho(\mathcal{A})-R_{\min }(\mathcal{A})+l}{\rho(\mathcal{A})-R_{\min }(\mathcal{A})+l(m-1) n},
\end{aligned}
$$

then

$$
\frac{x_{s}}{x_{t}} \leq\left\{\frac{\rho(\mathcal{A})-R_{\min }(\mathcal{A})+l}{l}\right\}^{\frac{1}{m-1}}
$$

On the other hand,

$$
\begin{aligned}
L \sum_{i_{2}, \ldots, i_{m}=1}^{n} x_{i_{2}} \cdots x_{i_{m}}-\rho(\mathcal{A}) x_{t}^{m-1} & =L \sum_{i_{2}, \ldots, i_{m}=1}^{n} x_{i_{2}} \cdots x_{i_{m}}-\sum_{i_{2}, \ldots, i_{m}=1}^{n} a_{t i_{2} \cdots i_{m}} x_{i_{2}} \cdots x_{i_{m}} \\
& \geq x_{t}^{m-1}\left[L(m-1) n-R_{\max }(\mathcal{A})\right]
\end{aligned}
$$

and

$$
\begin{aligned}
\frac{x_{t}^{m-1}}{\sum_{i_{2}, \ldots, i_{m}=1}^{n} x_{i_{2}} \cdots x_{i_{m}}} & =1-\frac{\sum_{i_{2}, \ldots, i_{m}=1}^{n} \begin{array}{c}
{\text { except } i_{2}=\cdots=i_{m}=t}_{i_{2}} \cdots x_{i_{m}} \\
\sum_{i_{2}, \ldots, i_{m}=1}^{n} x_{i_{2}} \cdots x_{i_{m}}
\end{array}}{\leq 1-\frac{[(m-1) n-1]}{\sum_{i_{2}, \ldots, i_{m}=1}^{n} x_{i_{2}} \cdots x_{i_{m}}} x_{s}^{m-1},}
\end{aligned}
$$


so we can get

$$
\begin{aligned}
\frac{x_{t}^{m-1}}{\sum_{i_{2}, \ldots, i_{m}=1}^{n} x_{i_{2}} \cdots x_{i_{m}}} & \leq \frac{L}{\rho(\mathcal{A})-R_{\max }(\mathcal{A})+L(m-1) n} \\
\frac{x_{s}^{m-1}}{\sum_{i_{2}, \ldots, i_{m}=1}^{n} x_{i_{2}} \cdots x_{i_{m}}} & \geq \frac{\rho(\mathcal{A})-R_{\max }(\mathcal{A})+[(m-1) n-1] L}{\rho(\mathcal{A})-R_{\max }(\mathcal{A})+L(m-1) n} \cdot \frac{1}{(m-1) n-1},
\end{aligned}
$$

then

$$
\frac{x_{s}}{x_{t}} \geq\left\{\frac{\rho(\mathcal{A})-R_{\max }(\mathcal{A})+[(m-1) n-1] L}{L} \cdot \frac{1}{(m-1) n-1}\right\}^{\frac{1}{m-1}} .
$$

This completes the proof.

In the following corollary, we get some bounds for $x_{t} / \sum_{j=1}^{n} x_{j}$ and $x_{s} / \sum_{j=1}^{n} x_{j}$ in terms of the entries of $\mathcal{A}$ and the spectral radius $\rho(\mathcal{A})$.

Corollary 2.4 If $\mathcal{A}$ is positive with Perron vector $x$. Then

$$
\begin{aligned}
& \frac{x_{t}}{\sum_{i_{k}=1}^{n} x_{i_{k}}} \geq \frac{l}{\rho(\mathcal{A})-R_{\min }(\mathcal{A})+n l} \\
& \frac{x_{s}}{\sum_{i_{k}=1}^{n} x_{i_{k}}} \leq \frac{\rho(\mathcal{A})-R_{\min }(\mathcal{A})+l}{\rho(\mathcal{A})-R_{\min }(\mathcal{A})+n l} \\
& \frac{x_{s}}{x_{t}} \leq \frac{\rho(\mathcal{A})-R_{\min }(\mathcal{A})+l}{l} .
\end{aligned}
$$

Proof From

$$
\rho(\mathcal{A}) x_{t}^{m-1}=\sum_{i_{2}, \ldots, i_{m}=1}^{n} a_{t i_{2} \cdots i_{m}} x_{i_{2}} \cdots x_{i_{m}} \geq\left(\sum_{i_{2}, \ldots, i_{m}=1}^{n} a_{t i_{2} \cdots i_{m}} x_{i_{k}}\right) x_{t}^{m-2}
$$

we have

$$
\begin{aligned}
\rho(\mathcal{A}) x_{t}-l \sum_{i_{k}=1}^{n} x_{i_{k}} & \geq \sum_{i_{2}, \ldots, i_{m}=1}^{n} a_{t i_{2} \cdots i_{m}} x_{i_{k}}-l \sum_{i_{k}=1}^{n} x_{i_{k}} \\
& \geq x_{t}\left[R_{\min }(\mathcal{A})-n l\right] .
\end{aligned}
$$

Similar to the proof of Theorem 2.3, we can get the results.

Remark Similar to the proof of Theorem 2.3, we can get the lower bound of $\frac{x_{s}}{x_{t}}$.

\section{Application to the perturbation bound}

Suppose $\mathcal{A} \geq 0$ and $\mathcal{B}$ is another tensor satisfying $\mathcal{A} \leq \mathcal{B}$. In this section we are interested in the bound of $\rho(\mathcal{B})-\rho(\mathcal{A})$.

Lemma 3.1 $[10] 0 \leq \mathcal{A} \leq \mathcal{B}$, then $\rho(\mathcal{A}) \leq \rho(\mathcal{B})$. 
We let $\mathbb{P}=\left\{\left(y_{1}, y_{2}, \ldots, y_{n}\right) \mid y_{i} \geq 0\right\}$ be the positive cone, and let the interior of $\mathbb{P}$ be denoted by $\operatorname{int}(\mathbb{P})=\left\{\left(y_{1}, y_{2}, \ldots, y_{n}\right) \mid y_{i}>0\right\}$.

Theorem 3.2 Let $\mathcal{A}$ be weakly irreducible. For a nonzero $x \in \mathbb{P}$, we define

$$
\mu(x)=\min _{x_{i}>0} \frac{\left(\mathcal{A} x^{m-1}\right)_{i}}{x_{i}^{m-1}}, \quad v(x)=\max _{x_{i}>0} \frac{\left(\mathcal{A} x^{m-1}\right)_{i}}{x_{i}^{m-1}} .
$$

Then

$$
\mu(x) \leq \rho(\mathcal{A}) \leq v(x)
$$

Proof Since

$$
\begin{aligned}
& \min _{x_{i}>0} \frac{\left(\mathcal{A} x^{m-1}\right)_{i}}{x_{i}^{m-1}} \leq \frac{\left(\mathcal{A} x^{m-1}\right)_{i}}{x_{i}^{m-1}} \leq \max _{x_{i}>0} \frac{\left(\mathcal{A} x^{m-1}\right)_{i}}{x_{i}^{m-1}}, \quad x_{i} \neq 0, \\
& \mu(x) x^{m-1} \leq \mathcal{A} x^{m-1} \leq v(x) x^{m-1} .
\end{aligned}
$$

By the result of Lemma 5.4 in [10], we have

$$
\mu(x) \leq \rho(\mathcal{A}) \leq v(x)
$$

Theorem 3.3 Let $\mathcal{A}, \mathcal{B}$ be irreducible and positive such that $\omega=\min _{i_{1}, \ldots, i_{m}}\left(b_{i_{1}, \ldots, i_{m}}-\right.$ $\left.a_{i_{1}, \ldots, i_{m}}\right) \geq 0$ and $\mu=\max _{i_{1}, \ldots, i_{m}}\left(b_{i_{1}, \ldots, i_{m}}-a_{i_{1}, \ldots, i_{m}}\right)$. Then

$$
\frac{\omega\left[\rho(\mathcal{A})-R_{\min }(\mathcal{A})+l(m-1) n\right]}{\rho(\mathcal{A})-R_{\min }(\mathcal{A})+l} \leq \rho(\mathcal{B})-\rho(\mathcal{A}) \leq \frac{\mu\left[\rho(\mathcal{A})-R_{\min }(\mathcal{A})+l(m-1) n\right]}{l} .
$$

Proof Let $x$ be the Perron vector of $\mathcal{A}$. Define $i$ as follows:

$$
\frac{\left(\mathcal{B} x^{m-1}\right)_{i}}{x_{i}^{m-1}}=\max _{k} \frac{\left(\mathcal{B} x^{m-1}\right)_{k}}{x_{k}^{m-1}} .
$$

Then, by Theorem 3.2, we have

$$
\max _{k} \frac{\left(\mathcal{B} x^{m-1}\right)_{k}}{x_{k}^{m-1}} \geq \rho(\mathcal{B})
$$

From the simple equality

$$
(\mathcal{B}-\mathcal{A}) x^{m-1}+\rho(\mathcal{A}) x^{[m-1]}=\mathcal{B} x^{m-1},
$$

and by considering the $i$ th coordinate,

$$
\frac{\sum_{i_{2}, \ldots, i_{m}=1}^{n}\left(b_{i i_{2} \cdots i_{m}}-a_{i i_{2} \cdots i_{m}}\right) x_{i_{2}} \cdots x_{i_{m}}}{x_{i}^{m-1}}+\rho(\mathcal{A})=\frac{\left(\mathcal{B} x^{m-1}\right)_{i}}{x_{i}^{m-1}} \geq \rho(\mathcal{B}) .
$$

Since $\mu=\max _{i_{1}, \ldots, i_{m}}\left(b_{i_{1}, \ldots, i_{m}}-a_{i_{1}, \ldots, i_{m}}\right)$ and $x_{t}=\min _{1 \leq i \leq n} x_{i}$,

$$
\rho(\mathcal{B})-\rho(\mathcal{A}) \leq \frac{\mu \sum_{i_{2}, \ldots, i_{m}=1}^{n} x_{i_{2}} \cdots x_{i_{m}}}{x_{t}^{m-1}} \leq \frac{\mu\left[\rho(\mathcal{A})-R_{\min }(\mathcal{A})+l(m-1) n\right]}{l} .
$$


Similarly, we can get

$$
\rho(\mathcal{B})-\rho(\mathcal{A}) \geq \frac{\omega \sum_{i_{2}, \ldots, i_{m}=1}^{n} x_{i_{2}} \cdots x_{i_{m}}}{x_{s}^{m-1}} \geq \frac{\omega\left[\rho(\mathcal{A})-R_{\min }(\mathcal{A})+l(m-1) n\right]}{\rho(\mathcal{A})-R_{\min }(\mathcal{A})+l} .
$$

Remark If we let $x$ be the Perron vector of $\mathcal{B}$, then by a similar method, we can get the following bound:

$$
\frac{\omega\left[\rho(\mathcal{B})-R_{\min }(\mathcal{B})+l(m-1) n\right]}{\rho(\mathcal{B})-R_{\min }(\mathcal{B})+l} \leq \rho(\mathcal{B})-\rho(\mathcal{A}) \leq \frac{\mu\left[\rho(\mathcal{B})-R_{\min }(\mathcal{B})+l(m-1) n\right]}{l} .
$$

\section{Conclusion}

We have obtained a new and sharper bound of $x_{s} / x_{t}$, lower bounds for $x_{t}^{m-1} / \sum x_{i_{2}} \cdots x_{i_{m}}$, and upper bounds for $x_{s}^{m-1} / \sum x_{i_{2}} \cdots x_{i_{m}}$, where $x=\left(x_{i}\right)>0$ is the Perron vector of a positive tensor $\mathcal{A}$ with $x_{s}=\max _{1 \leq i \leq n} x_{i}$ and $x_{t}=\min _{1 \leq i \leq n} x_{i}$. In addition we have given bounds of $\rho(\mathcal{B})-\rho(\mathcal{A})$ when $0<\mathcal{A} \leq \mathcal{B}$ by these bounds.

\section{Competing interests}

The authors declare that they have no competing interests.

\section{Authors' contributions}

All authors contributed equally to this work. All authors read and approved the final manuscript.

\section{Acknowledgements}

This research is supported by NSFC (61170311), Chinese Universities Specialized Research Fund for the Doctoral Program (20110185110020), Sichuan Province Sci. \& Tech. Research Project (12ZC1802). The first author is supported by the Fundamental Research Funds for Central Universities.

\section{Received: 26 March 2014 Accepted: 14 August 2014 Published: 03 Sep 2014}

\section{References}

1. Chang, KC, Pearson, K, Zhang, T: Primitivity, the convergence of the NZQ method, and the largest eigenvalue for nonnegative tensors. SIAM J. Matrix Anal. Appl. 32, 806-819 (2011)

2. Qi, L: Eigenvalues and invariants of tensor. J. Math. Anal. Appl. 325, 1363-1377 (2007)

3. Ng, M, Qi, L, Zhou, G: Finding the largest eigenvalue of a non-negative tensor. SIAM J. Matrix Anal. Appl. 31, 1090-1099 (2009)

4. Chang, KC, Zhang, T: Perron-Frobenius theorem for nonnegative tensors. Commun. Math. Sci. 6, 507-520 (2008)

5. Chang, KC, Pearson, K, Zhang, T: On eigenvalue problems of real symmetric tensors. J. Math. Anal. Appl. 350, 416-422 (2009)

6. Liu, Y, Zhou, G, Ibrahim, NF: An always convergent algorithm for the largest eigenvalue of an irreducible nonnegative tensor. J. Comput. Appl. Math. 235, 286-292 (2010)

7. Zhou, G, Caccetta, L, Qi, L: Convergence of an algorithm for the largest singular value of a nonnegative rectangular tensor. Linear Algebra Appl. 438, 959-968 (2013)

8. Qi, L: Eigenvalues of a real supersymmetric tensor. J. Symb. Comput. 40, 1302-1324 (2005)

9. Lim, LH: Singular values and eigenvalues of tensors: a variational approach. In: Proceedings of the IEEE International Workshop on Computational Advances in Multi-Sensor Adaptive Processing, CAMSAP 05, pp. 129-132 (2005)

10. Yang, Y, Yang, Q: Further results for Perron-Frobenius theorem for nonnegative tensors. SIAM J. Matrix Anal. Appl. 31 , 2517-2530 (2010)

11. Yang, Y, Yang, Q: Further results for Perron-Frobenius theorem for nonnegative tensors II. SIAM J. Matrix Anal. Appl. 32, 1236-1250 (2011)

12. Wen, $\mathrm{L}, \mathrm{Ng}, \mathrm{M}$ : The perturbation bound for the spectral radius of a non-negative tensor. http://www.math.hkbu.edu.hk/mng/tensor-research/tensor

10.1186/1029-242X-2014-340

Cite this article as: He et al:. Some inequalities for nonnegative tensors. Journal of Inequalities and Applications 2014, 2014:340 